\title{
Oblique angled plasma etching for 3D silicon structures with wiggling geometries
}

\section{Chang, Bingdong}

Published in:

Nanotechnology

Link to article, DOI:

10.1088/1361-6528/ab53fb

Publication date:

2019

Document Version

Peer reviewed version

Link back to DTU Orbit

Citation (APA):

Chang, B. (2019). Oblique angled plasma etching for 3D silicon structures with wiggling geometries.

Nanotechnology, 31(8), [085301]. https://doi.org/10.1088/1361-6528/ab53fb

\section{General rights}

Copyright and moral rights for the publications made accessible in the public portal are retained by the authors and/or other copyright owners and it is a condition of accessing publications that users recognise and abide by the legal requirements associated with these rights.

- Users may download and print one copy of any publication from the public portal for the purpose of private study or research.

- You may not further distribute the material or use it for any profit-making activity or commercial gain

- You may freely distribute the URL identifying the publication in the public portal

If you believe that this document breaches copyright please contact us providing details, and we will remove access to the work immediately and investigate your claim 
ACCEPTED MANUSCRIPT

\section{Oblique angled plasma etching for 3D silicon structures with wiggling geometries}

To cite this article before publication: Bingdong Chang 2019 Nanotechnology in press https://doi.org/10.1088/1361-6528/ab53fb

\section{Manuscript version: Accepted Manuscript}

Accepted Manuscript is "the version of the article accepted for publication including all changes made as a result of the peer review process, and which may also include the addition to the article by IOP Publishing of a header, an article ID, a cover sheet and/or an 'Accepted

Manuscript' watermark, but excluding any other editing, typesetting or other changes made by IOP Publishing and/or its licensors"

This Accepted Manuscript is @ 2019 IOP Publishing Ltd.

During the embargo period (the 12 month period from the publication of the Version of Record of this article), the Accepted Manuscript is fully protected by copyright and cannot be reused or reposted elsewhere.

As the Version of Record of this article is going to be / has been published on a subscription basis, this Accepted Manuscript is available for reuse under a CC BY-NC-ND 3.0 licence after the 12 month embargo period.

After the embargo period, everyone is permitted to use copy and redistribute this article for non-commercial purposes only, provided that they adhere to all the terms of the licence https://creativecommons.org/licences/by-nc-nd/3.0

Although reasonable endeavours have been taken to obtain all necessary permissions from third parties to include their copyrighted content within this article, their full citation and copyright line may not be present in this Accepted Manuscript version. Before using any content from this article, please refer to the Version of Record on IOPscience once published for full citation and copyright details, as permissions will likely be required. All third party content is fully copyright protected, unless specifically stated otherwise in the figure caption in the Version of Record.

View the article online for updates and enhancements. 


\title{
Oblique angled plasma etching for 3D silicon structures with wiggling geometries
}

\section{Bingdong Chang}

DTU Nanolab, Technical University of Denmark, Ørsteds Plads, 2800 Kgs. Lyngby, Denmark.

E-mail: bincha@dtu.dk

Received xxxxxx

Accepted for publication $\mathrm{xxxxxx}$

Published xxxxxx

\begin{abstract}
Three dimensional silicon micro- and nanostructures have attracted special research interest, particularly in photonic and electrochemical devices, due to the extra degrees of freedom for manipulation of device performance and properties. However, it is still considered to be difficult to fabricate three dimensional silicon structures with an arbitrary geometric form in a scalable volume, especially with the standard fabrication techniques, which are intrinsically directional and anisotropic. In this work we proposed a unique method of oblique-angled plasma etching from various angles, thus multilayered silicon structures with wiggling geometries can be fabricated in a controllable manner both in micro- and nanoscale. The mechanism is explained as the induced modifications of substrate topology and surface charging when a glass pad is attached on the sample surface, thus the incoming ion fluxes can be directed to the substrate surface with an off-normal angle. The process is convenient to perform without additional modifications on the plasma etching systems. In the same time, it provides more possibilities in the toolkit for fabricating three dimensional silicon structures with conventional fabrication technologies.
\end{abstract}

Keywords: DRIE, DREM, 3D fabrication, oblique-angled plasma etching

\section{Introduction}

Three dimensional (3D) engineering in both micro- and nanoscale has attracted increasing research interests nowadays. Ideally, by shaping structures in arbitrary geometric forms in a scalable volume, advanced devices can be fabricated and sophisticated functions can be realized compared with standard structures in a two dimensional manner. However, due to the high directionality of energetic particles like ions, electrons and laser-generated photons, most of the standard fabrication techniques in semiconductor industries (as lithography, plasma etching and physical vapor deposition) are intrinsically confined in one direction, thus an anisotropic structure is normally created by default. In order to add features to structures in all 3 dimensions, either modification should be made based on existing techniques, or unorthodox technologies have to be introduced $[1,2]$. Here we will focus the discussion on plasma etching-based $3 \mathrm{D}$ processes on silicon materials.

Since most of the positively charged ions (as $\mathrm{SF}_{5}{ }^{+}$and $\mathrm{Ar}^{+}$) are important etching species in a plasma process, and the ion trajectories are directed by the electric field within the ion sheath, which is normally conformal to the substrate surface, thus the etching process will typically proceed in a direction normal to the sample surface. In order to give a degree of freedom to incoming ions on the off-normal directions, a popular method is to install a Faraday cage inside the plasma etching chamber, thus the ion trajectories 


\begin{tabular}{ccccccc}
\hline & \multicolumn{3}{c}{ DREM 0.5kW } & \multicolumn{2}{c}{ DREM 2kW } \\
\cline { 2 - 7 } & Deposition & removal & Etch & Deposition & Removal & Etch \\
\hline Time $(\mathrm{s})$ & 3.2 & 1.0 & 1.5 & 2.5 & 1.0 & 2.0 \\
\hline $\mathrm{C}_{4} \mathrm{~F}_{8}$ gas flow $(\mathrm{sccm})$ & 25 & 5 & 5 & 150 & 10 & 10 \\
\hline $\mathrm{SF}_{6}$ gas flow $(\mathrm{sccm})$ & 10 & 10 & 30 & 15 & 100 & 100 \\
\hline Argon gas flow $(\mathrm{sccm})$ & 100 & 75 & 100 & 2000 & 2000 & 2000 \\
\hline Coil power $(\mathrm{W})$ & 500 & 500 & 500 & 0 & 100 & 0 \\
\hline Platen power $(\mathrm{W})$ & 0 & 40 & 0 & 20 & 3 & 20 \\
\hline Pressure $(\mathrm{mTorr})$ & 10 & 1.8 & 10 & -19 & -19 & -19 \\
\hline Temperature $\left({ }^{\circ} \mathrm{C}\right)$ & -19 & -19 & -19 & 200 & 19 \\
\hline
\end{tabular}

Table 1. An overview of different parameter settings for etching micro- and nanostructures (It should be noted that helium cooling on the wafer backside was used to control the wafer temperature, and the helium cooling pressure was set to be at 10 Torr in all processes).

can be modified and incident ions can reach the substrate with an off-normal angle according to the sample surface, thus etching process can proceed in an oblique direction [3, $4,5]$. The incident angle of incoming ions can also be tuned with a tilted sample loading position [6, 7] or a shadow mask [8], and structures with a maximum tilted angle of around $70^{\circ}$ can be reached. Another method is to making use of the isotropicity of electrically neutral radicals, which can give an isotropic etch profile, thus suspended structures or even $3 \mathrm{D}$ periodic structures can be fabricated conveniently. [9] With modified plasma etching techniques as discussed above, different kinds of 3D silicon structures have been fabricated for applications like 3D photonic crystals [10, 11, 12], slanted nanohairs [13], photocatalysis [14], etc.

Here we report a novel and facile fabrication method for 3D silicon micro- and nanostructures with a wiggling geometry. By attaching a piece of glass pad close to the pattern region, a charging effect can be induced on the surface of the glass pads, which will direct the incoming ion fluxes with positive charges onto the substrate with an offnormal angle. Depending on the thickness of the glass pad, the tilted angle can be as large as $12^{\circ}$. By modifying the relative position between the glass pad and the pattern area and performing plasma etching multiple times, a multilayered silicon structure can be created with a wiggling geometry, with each layer having a distinctive tilted angle compared with substrate surface. The etching process is performed with a DREM (Deposit, Remove, Etch Multistep) process to insure a good sidewall protection and etching directionality [15]. Compared with previous research, this method doesn't require additional setups inside the etching system, thus contaminations from metals or sputtered micromasks can be avoided. Besides, novel properties could be interesting to investigate with this new type of structures with a wiggling geometry.

\section{Materials and Methods}

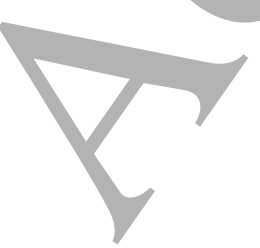

Depending on the critical dimension of patterns, various lithography techniques have been performed, and the substrate used were 4-inch silicon wafers (single side polished, $\langle 100\rangle$ crystalline orientation). Microstructures are patterned with maskless UV lithography (MLA100, Heidelberg Instrument), which is a direct writing system equipped with $365 \mathrm{~nm}$ LED light source, the resist we used was negative tone photoresist AZ nLOF 2020 (MicroChemicals) with a thickness of $1.5 \mu \mathrm{m}$, and the dose applied was $220 \mathrm{~mJ} / \mathrm{cm}^{2}$.

For $200 \mathrm{~nm}$ dot patterns, deep UV stepper lithography was performed to realize a large writing area with high throughputs. Silicon wafers were coated with $65 \mathrm{~nm}$ thick bottom antireflective coating (BARC, DUV42s-6, Brewer Science)/layer and a $360 \mathrm{~nm}$ thick DUV resist (JSR KRF M230Y, JSR-Micro). Afterwards dots with diameter of 200 nm were patterned by a DUV stepper system (FPA$3000 \mathrm{EX} 4$, Canon), which was equipped with a $248 \mathrm{~nm} \mathrm{KrF}$ excimer (intensity of $2800 \mathrm{~W} / \mathrm{cm}^{2}$ ). The dose we used was 21 $\mathrm{mJ} / \mathrm{cm}^{2}$ and the focus depth was set to be $0.17 \mu \mathrm{m}$. The samples were baked after exposure at $130{ }^{\circ} \mathrm{C}$ and developed in AZ726 (AZ Electronic Materials), rinsed in deionized water.

For nanostructures with critical dimensions below 100 $\mathrm{nm}$, electron beam lithography was applied with a $100 \mathrm{kV}$ electron beam writing system (Jeol JBX-9500FS, JEOL). Positive tone e-beam resist AR-P 6200.04 (ALLRESIST $\mathrm{GmbH}$ ) was used to achieve a thin resist layer with thickness of $85 \mathrm{~nm}$, thus pattern collapse could be avoided for small dimensions. During the exposure current was set at $6 \mathrm{nA}$ and dose at $260 \mu \mathrm{C} / \mathrm{cm}^{2}$. Exposed samples were developed with AR 600-54/8 (ALLRESIST GmbH) and rinsed with isopropanol.

All the plasma etching processes were performed with an inductively coupled plasma (ICP) etching system (DRIE Pegasus, SPTS). Depending on the critical dimension of the structures, two sets of parameters have been used for etching process: DREM $0.5 \mathrm{~kW}$ is a mild process to create scallop size of around $20 \mathrm{~nm}$, thus is used for etching nanostructures, 


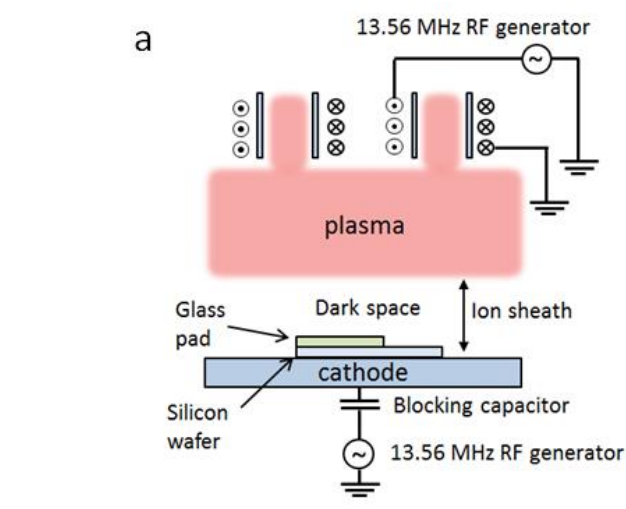

b
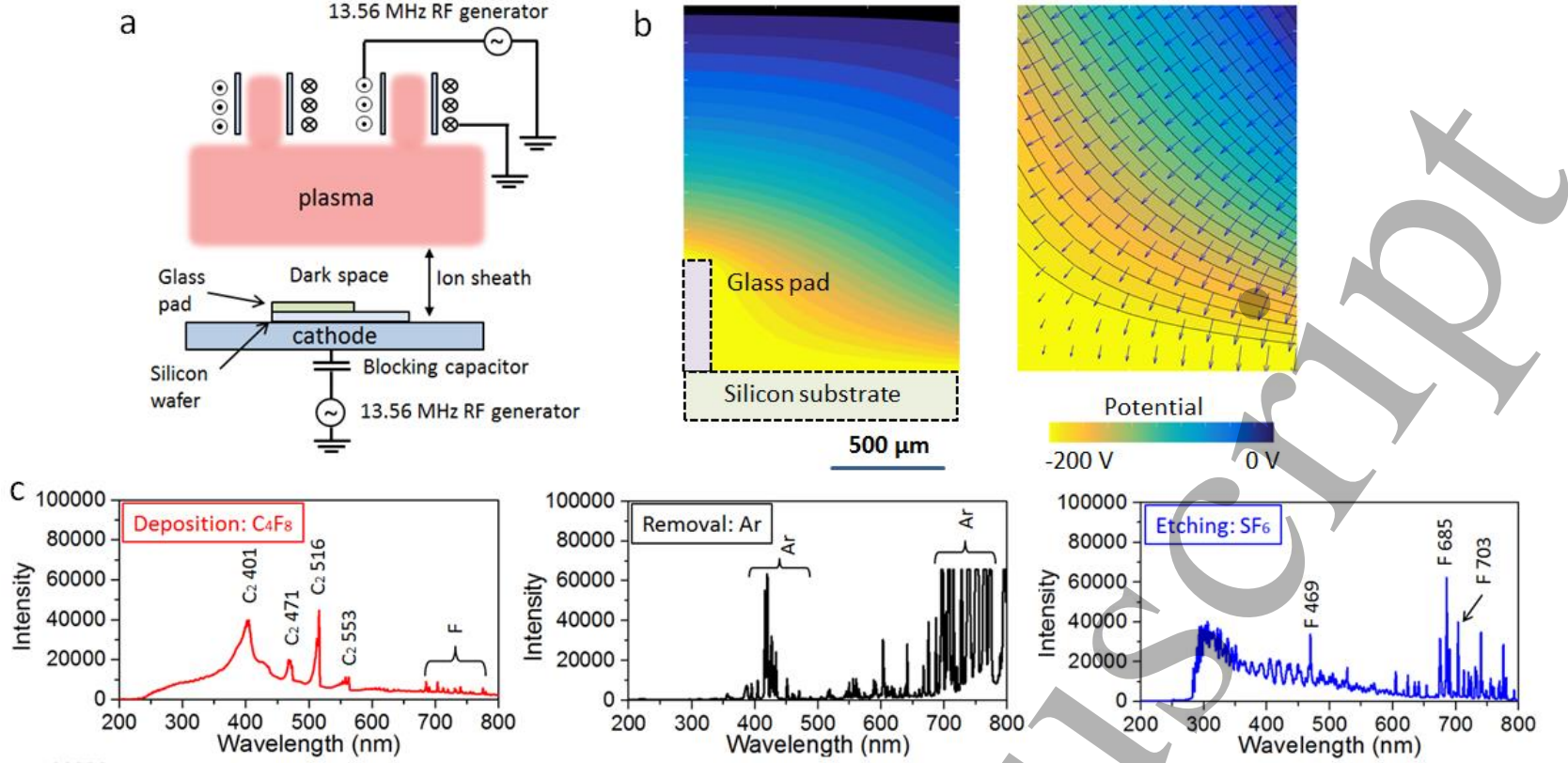

Figure 1. (a) A simple illustration of the etching system and the experiment setup; (b) Simulated ion sheath potential distribution and electric field near the interface between glass pad and silicon substrate; (c) Optical emission spectra from different chemical species during a 3-step DREM process.

while DREM $2 \mathrm{~kW}$ has a faster etch rate with scallop size of around $200 \mathrm{~nm}$, which is suitable for etching microstructures.

The samples were diced manually into small pieces (with the size of around $1.5 \mathrm{~cm}$ by $1.5 \mathrm{~cm}$ ) and attached on a carrier wafer (4 inch, single side polished with around $5 \mu \mathrm{m}$ thick silicon oxide layer grown by thermal oxidation). Before performing DREM process on the samples, oxygen plasma was applied to remove the BARC layer or the residues after lithography. The glass pad we used was diced manually from a 4 inch borofloat wafer with thickness of $500 \pm 10 \mu \mathrm{m}$, the size of the glass pad is comparable with the sample pieces. Crystalbond adhesives were used to attach the glass pad onto the sample surface. The etched samples are inspected with scanning electron microscope (SEM). Equipotential lines and ion trajectories within the ion sheath are simulated with Matlab (R2015b, MathWorks).

\section{Results and Discussions}

Figure 1(a) shows a schematic diagram of the ICP etching system used in the experiments. Plasma is generated with a dual-coil 13.56 MHz radio frequency (RF) generator, and ions are accelerated by a potential bias with the power supplied by a $13.56 \mathrm{MHz}$ platen RF generator. During etching processes, samples are electrostatically clamped onto the platen, which is electrically performing as a cathode. Due to the good electrical conductivity of ionized gases, there is almost no potential drop inside the plasma. However, a plasma sheath is formed close to the sample surface, giving a concentrated local electric field with a thickness of around few millimeters. Inside the plasma sheath, positive ions can be accelerated and arrive the sample surface with high kinetic energy and narrow distribution of incident angles. Normally the ion sheath is conformal to the sample surface, since samples in standard processes are 2D planar surface in geometry, ions will bombard the surface vertically and etching process will be anisotropic. However, it has been realized before that a local sheath curvature can be induced because of substrate topography $[16,17]$ or charging effect $[18,19]$, e.g. from an etching mask, thus the ion trajectories can be distorted and ion incoming angles will be off-normal compared to the sample surface. In order to adding 3D features to the silicon structures by directing ion fluxes, a glass pad is attached on top of silicon surface close to the pattern area as shown in figure 1(a). Since the electrons have a wider angular distribution compared with ions, there will be more electrons accumulated on glass surface compared with the silicon substrate. More importantly, glass is a poor electrical conductor, which will make the surface of glass pad stay negatively charged. Considering about these factors, a simulation of potential field in the plasma sheath is performed by solving the Laplace's equation [16] as shown in figure 1(b), the potential on the substrate surface is assumed to be $-200 \mathrm{~V}$, which is read during the etching process. On top of the glass surface a larger potential gradient is observed compared with the silicon surface, which is due to a higher charge density on top of the glass pad. The imparity of surface charging between glass and 
silicon, together with the surface topology, will give a distortion of ion trajectories close to the step region near glass-silicon interface, which can be seen in the simulated electric field distribution. While part of the incoming ion flux will be accelerated and directed to the sidewall of the glass pad, part of the ions will arrive on the silicon surface with a tilted angle, thus the etching process will proceed in an offnormal angle. If a conducting material is used instead of glass, free electrons will be able to compensate for the local charging imbalance, and the effect for ion trajectory distortion will be limited. It should also be mentioned that by using glass pads, the loading area of silicon will not be affected, thus the etch rate will not be reduced due to the loading effect. [20]

The etching process used in the experiments is a 3-step switched process named as DREM process, which is a modified Bosch process [15]. Briefly speaking, DREM process applies $\mathrm{C}_{4} \mathrm{~F}_{8}$ as the passivation gas without bias, thus an isotropic, sufficient sidewall protection can be achieved; afterwards, the deposited fluorocarbon layer is removed by argon (Ar) plasma with platen power applied; the exposed silicon surface is then free to be etched with $\mathrm{SF}_{6}$ gas without platen power, thus the scallop size can be minimized. The DREM sequence will proceed and silicon will be etched continuously, the optical emission spectra of chemical species in different steps can be seen in figure 1(c). There are a few benefits to use DREM process for oblique-angled plasma etching: firstly, $\mathrm{Ar}^{+}$is the only positively charged ion species that is accelerated and directed by the local electric field, since $\mathrm{Ar}^{+}$has a smaller weight compared with other positive ions like $\mathrm{SF}_{5}^{+}$, a larger deflection of ion fluxes is possible for a bigger tilted angle; secondly, a low processing pressure can be achieved with Ar plasma ( 2 mTorr is possible depending on the gas flow and coil power), thus less ion collisions will happen, giving a good etching directionality; thirdly, a good sidewall passivation is realized with isotropic fluorocarbon deposition, this is essential for creating multilayered 3D structures, which will be discussed in detail later.

It can also be noticed from the simulated electric field, that the ion sheath curvature is less significant when it is far away from the glass pad. In order to understand the effective distance, in which ion incoming angles can be directed, we performed oblique-angled plasma etching process on silicon chips with patterns of $200 \mathrm{~nm}$ dots. 100 cycles of DREM $0.5 \mathrm{~kW}$, giving a total etch depth of around $2 \mu \mathrm{m}$. A glass pad is attached on top of the chip, and the tilted angle is measured by SEM as a function of distance between the measured pillar and the edge of glass pad (here the tilted angle is defined as the angle between the incoming ion trajectory and the normal direction of the substrate surface), results are shown in figure $\mathbf{2 ( a )}$. We can see that for a glass pad with a thickness of around $1000 \mu \mathrm{m}$, a larger tilted angle of $13^{\circ}$ can be achieved close to the edge of glass pad, compared with a maximum of around $8^{\circ}$ with a $500 \mu \mathrm{m}$ thick glass pad, this can be explained as a larger distortion of local electric field when a thicker glass pad is applied. The difference can also be seen in the SEM images at position 1 and 1' in figure 2(b). For pillars with an increasing distance from the edge of the glass pad, measured tilted angle will decrease monotonically for glass pad with thickness of both $500 \mu \mathrm{m}$ and $1000 \mu \mathrm{m}$, figure 2(b) shows the SEM images of pillars with a distance of $4 \mathrm{~mm}$ to the edge of glass pads (position 2 and 2'). This distribution of tilted angles is in agreement with previous research, where a silicon step is used [17], or the mask is charged with different surface

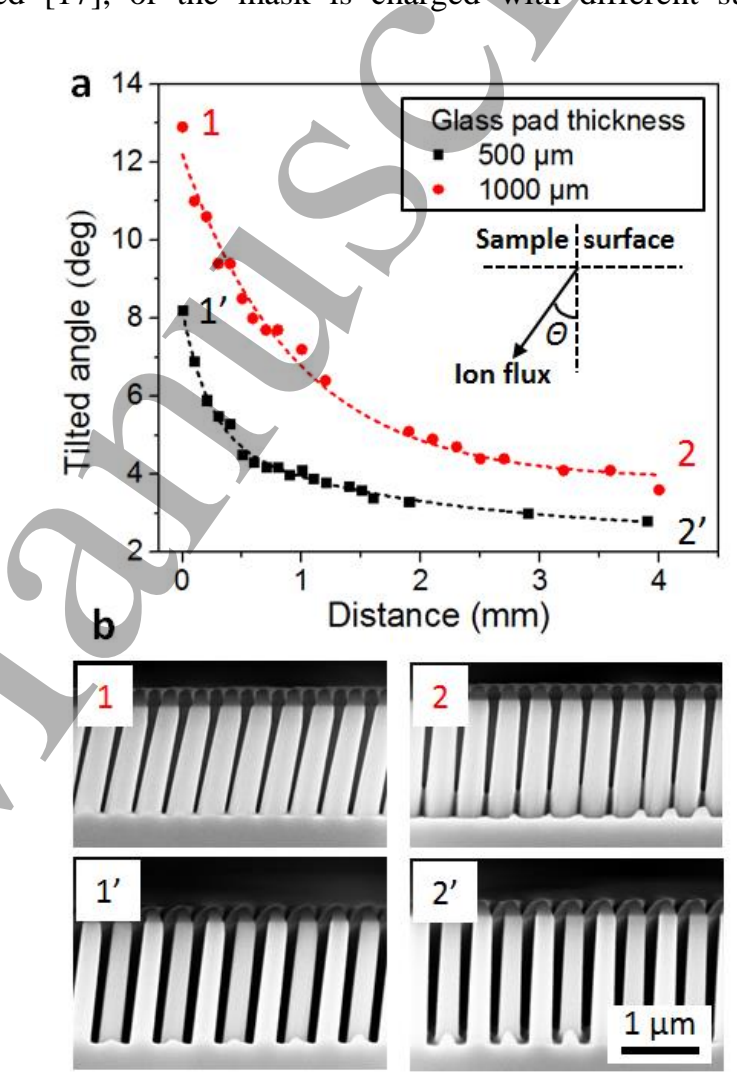

Figure 2. (a) Measured tilted angles of $250 \mathrm{~nm}$ nanopillar structures as a function of distance from the edge of glass pads; (b) SEM images of tilted nanopillar structures at different positions as illustrated in (a).

potentials. [21] In our experiments, the distance between the glass pad and pattern region is controlled to be within $2 \mathrm{~mm}$, in order to have an effective tuning of structure profiles. It should also be mentioned that the etch depth will decrease for the pillars close to the edge of glass pad (as shown in figure S1), this is due to the reduced local potential gradient in the corner area between the glass pad and the silicon substrate, which is illustrated in the simulated electric field in figure 1(b).

By exploiting the oblique-angled plasma etching, 3D silicon structures can be fabricated both in micro- and 

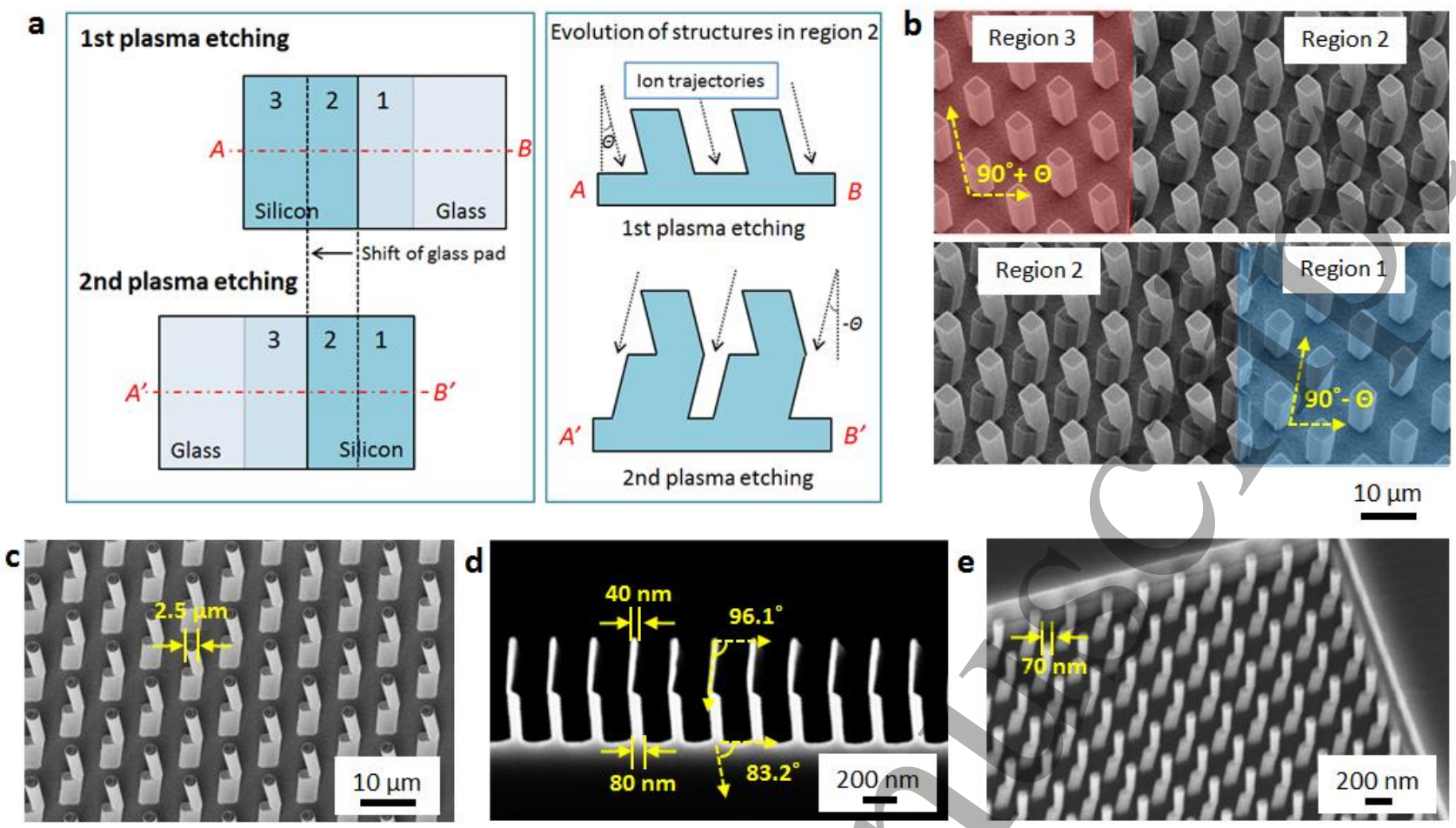

Figure 3. Creating two-layered 3D silicon structures, each layer has a different tilted angle. (a) An illustration of the process flow and structure evolution from a cross section view; (b) SEM images of micropillar structures in three different regions as shown in (a); and SEM images of different micro- and nanostructures, including (c) micropillar arrays; (d) silicon nanofin structures with linewidth of $40 \mathrm{~nm}$ and (d) nanopillar arrays with a pattern diameter of around $70 \mathrm{~nm}$.

nanoscale. Figure 3(a) shows the process flow for fabricating double layered silicon structures with different tilted angles on two layers. During the first time plasma etching, a glass pad is placed on silicon chip over region 1, thus tilted structures can be created in both region 2 and part of region 3 with a tilted angle of $\theta$, while region 1 will not be etched. The sample is then retrieved from the etching machine and the glass pad is moved manually from region 1 to region 3, followed by a second time plasma etching, during which structures in region 2 and part of region 1 will be etched but with a different tilted angle of $-\theta$. It can be seen that structures in region 2 is etched twice, each time with a different tilted angle, the profile evolution is illustrated in figure 3(a). There are a few issues that need to be addressed: firstly, during second time plasma etching, a step-like profile will be generated on the second layer, which is due to the shadow effect from the tilted first layer and the oblique-angled incoming ions, thus the dimension of the second layer will be enlarged compared with first layer; secondly, part of the sidewall on the first layer is suffering from more ion bombardment during the second time etching, however, due to the fluorocarbon protection on the sidewall, the first layer can still remain intact, this is benefited from the sufficient $\mathrm{C}_{4} \mathrm{~F}_{8}$ deposition in DREM process; thirdly, 500 $\mu \mathrm{m}$ thick glass pad is used to have a small tilted angle of around $6^{\circ}$, thus direct ion bombardment can be avoided to protect the sidewall of structures.

Figure 3(b) shows the SEM images of silicon microstructures with square patterns in different regions, we can see that structures in region 1 and 3 have opposite tilting angles, while double-layered structures are created in region 2. In figure 3(c) are arrays of double layered silicon pillars, with a pattern diameter of around $2.5 \mu \mathrm{m}$. Each of the layers for microstructures is created with 50 cycles of DREM $2 \mathrm{~kW}$ process, giving an etch depth of around $10 \mu \mathrm{m}$ on each layer. In figure $3(\mathrm{~d})$ and figure $3(\mathrm{e})$ are double-layered silicon nanostructures. Figure 3(d) shows a cross section view of silicon nanofin structures with a pattern width of $40 \mathrm{~nm}$, we can see the opposite tilted angles on the two layers $\left(6.1^{\circ}\right.$ on first layer and $-6.8^{\circ}$ on the second layer), and the second layer has a larger linewidth of around $80 \mathrm{~nm}$. Double layered silicon nanopillars are also fabricated with a diameter of 70 $\mathrm{nm}$, as shown in figure 3(e). For the nanostructures, 12 cycles of DREM $0.5 \mathrm{~kW}$ cycles are performed on each layer.

By repeating the oblique-angled plasma etching for multiple times, 3D silicon structures with wiggling geometries can be fabricated. Following the discussion above, figure 4(a) shows the structure profile evolution

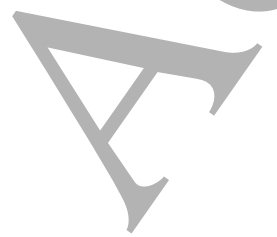


a Ion trajectories for 3rd time plasma etching $\mathbf{b}$
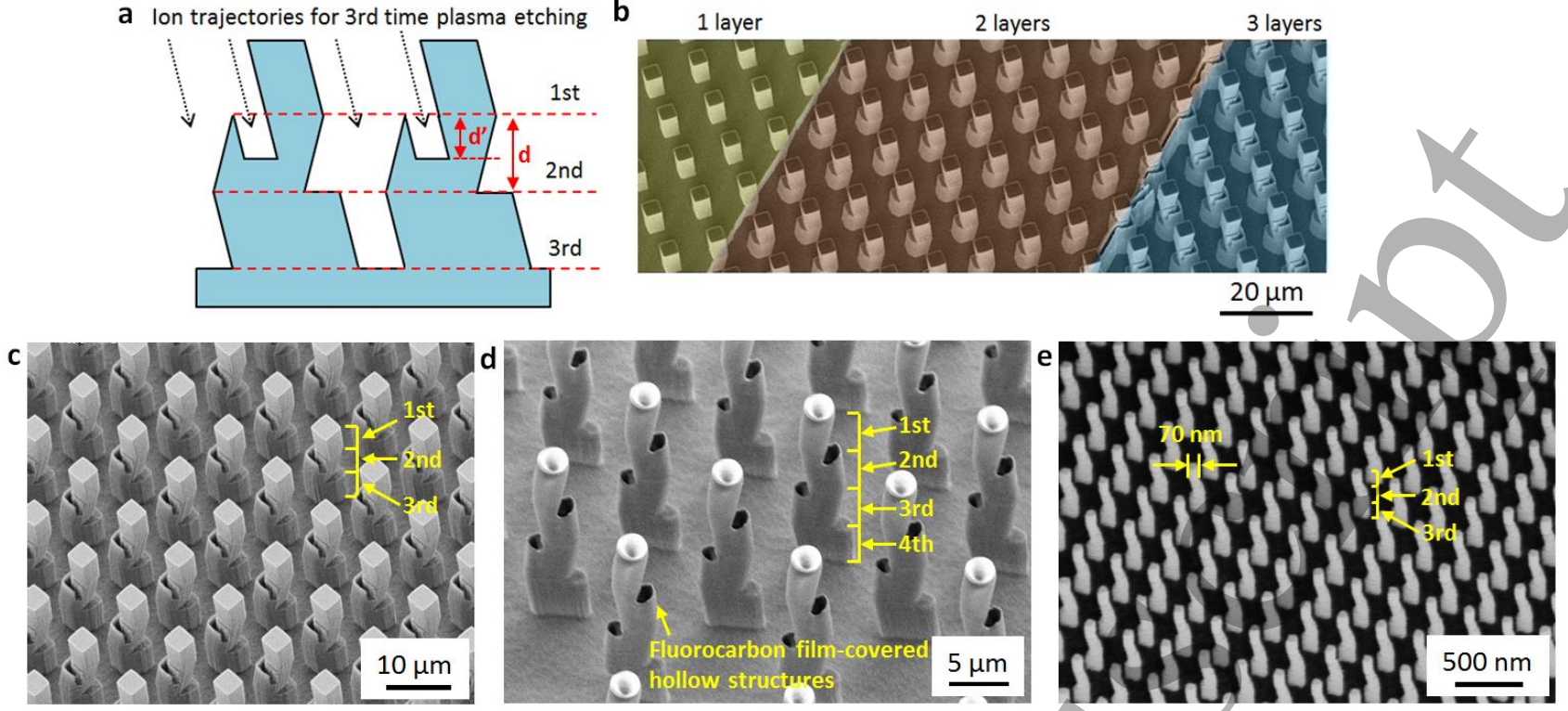

Figure 4. Creating 3D silicon structures with wiggling geometries by more than two times of plasma etching: (a) An illustration of profile evolution during a third time plasma etching following the previous process as in figure 3(a); (b) SEM image of three types of structures with different number of layers on a single chip; (c) SEM image of micropillar arrays with 3 layers; (d) Micropillar arrays with 4 layers; (e) Nanopillar arrays with 3 layers.

during a third time plasma etching, when the glass pad is placed back to the origin position as in the first time etching process. Since the tilted angle on the third layer is the same as the first layer, 3D structures with a wiggling geometry can be created. Again, due to the shadow effect from the first layer, a step-like structure will be created on the third layer (just as during the second time plasma etching), giving an even larger structure width on the third layer. Another important issue to notice is the profile change on the second layer, since the ion incoming angles are the same during first and third plasma etching, the step-like structure on the second layer will be under direct ion bombardment. Since a fluorocarbon film is deposited uniformly over the whole structure (with a thickness of around $50 \mathrm{~nm}$ ), which is chemically inert to fluorine-based etching species, the etching on the second layer will be delayed, thus the etch depth d' on the step structure is smaller compared with the height of second layer $d$, and the structure collapse can be avoided. Figure 4(b) shows different types of microstructures created on the single chip during multiple times of plasma etching, the edges between the different structure regions are caused by displacements of the glass pad.

More SEM images are shown in figure 4(c-e). Figure 4(c) shows arrays of silicon micropillars with 3 layers, we can see the gap-like profile on the second layer caused by the third time plasma etching as discussed earlier. Figure $4(d)$ is a SEM image of 4 layered silicon micropillars, which is created with 4 times of oblique-angled etching, while the first layer and third layer have the same tilted angle, the fourth layer has the same tilted angle as the second layer, thus giving a wiggling geometry to the structures. We can notice the shell-like hollow structures on the sidewall of micropillars, this is again due to the etching of the step-like structures on both second and third layer. Since the fluorocarbon film is not removed after etching process, which is free from etching process, hollow structures will be created with a shell of fluorocarbon film (SEM images of the structures after plasma ashing is shown in figure S2, in which fluorocarbon film is removed by oxygen plasma, thus the hollow structure is destroyed). Each layer of the structures in figure $4(\mathrm{c})$ and figure $4(\mathrm{~d})$ is created with 30 cycles of DREM $2 \mathrm{~kW}$ process. The multi-angled plasma etching can also be performed on nanostructures, which is shown in figure 4(e), where nanopillars with a diameter of 70 $\mathrm{nm}$ are etched with three times oblique-angled plasma process ( 8 cycles of DREM $0.5 \mathrm{~kW}$ process). Because of the small critical dimension, the step-like profile and hollow structures are almost unnoticeable, giving a smooth wigging structure profile. It should be noticed that metal-assisted chemical etching can also be used to create tilted or even zigzag-like silicon structures [22, 23], while to create wiggling silicon structures with plasma etching techniques hasn't been reported before. By making use of the fluorocarbon/silicon-based hollow structures (and possibly an additional process of fluorocarbon film deposition to enclose the microcavities), it can be promising to fabricate microcontainers or reservoirs for biological or medical applications. [24, 25] It should be mentioned that the alignment between the glass pad and the sample surface is 
performed manually during the process. In order to have a more accurate control of the relative position between glass pad and the sample, some simple setups can be designed, which is however beyond the scope of this paper.

\section{Conclusions}

In this paper, we introduced an unconventional fabrication method for multilayered 3D silicon structures in both microand nanoscale, and each of the layers can have a distinctive tilted angle compared with the substrate surface, which is enabled by oblique-angled plasma etching from different angles. The mechanism and method are discussed for oblique-angled plasma etching process, which is based on modifications of surface charges and substrate topology by using of a glass pad, thus incoming ion fluxes can arrive at the surface with an off-normal angle (up to $13^{\circ}$ ). Different types of 3D structures up to 4 layers have been fabricated, showing some intriguing geometric features like wiggling profiles and hollow structures on the sidewalls. Such kind of asymmetric structures, especially in nanoscale, could be promising to give some novel insights and applications in nano-optics and nanomechanics, e.g. higher order optical diffraction from asymmetric gratings, biomimicking gecko setae with asymmetric nanopillar structures, etc.

\section{Acknowledgements}

The author would like to thank the DTU Nanolab staff for instrument support and Dr. Jörg Hübner for fruitful discussions. This work was supported by a research grant (00027987) from VILLUM FONDEN.

\section{References}

[1] Tawfick S, De Volder M, Copic D, Park S J, Oliver C R, Polsen E S, Roberts M J and Hart A J 2012. Engineering of micro-and nanostructured surfaces with anisotropic geometries and properties Adv. Mater. 24(13) 1628-1674

[2] Seniutinas G, Balčytis A, Reklaitis I, Chen F, Davis J, David C and Juodkazis S 2017 Tipping solutions: emerging 3D nano-fabrication/-imaging technologies Nanophotonics 6(5) 923-941

[3] Lee J K, Lee S H, Min J H, Jang I Y, Kim C K and Moon S H 2009 Oblique-directional plasma etching of Si using a Faraday cage J. Electrochem. Soc. 156(7) D222-D225

[4] Bruce Burckel D, Finnegan P S, David Henry M, Resnick P $\mathrm{J}$ and Jarecki Jr RL 2016 Oblique patterned etching of vertical silicon sidewalls Appl. Phys. Lett. 108(14) 142103

[5] Latawiec P, Burek M J, Sohn Y I and Lončar M 2016 Faraday cage angled-etching of nanostructures in bulk dielectrics J. Vac. Sci. Technol. B 34(4) 041801

[6] Cybart S A, Roediger P, Ulin-Avila E, Wu S M, Wong T J and Dynes R C 2013 Nanometer scale high-aspect-ratio trench etching at controllable angles using ballistic reactive ion etching J. Vac. Sci. Technol. B 31(1) 010604
[7] Takamori T, Coldren L A and Merz J L 1988 Angled etching of $\mathrm{GaAs} / \mathrm{AlGaAs}$ by conventional $\mathrm{Cl} 2$ reactive ion etching Appl. Phys. Lett. 53(25) 2549-2551

[8] Jacobs B and Zengerle R 1996 Reactive ion etching of sloped sidewalls for surface emitting structures using a shadow mask technique J. Vac. Sci. Technol. B 14(4) 2537-2542.

[9] Chang B, Jensen F, Hübner J and Jansen H 2018 DREM2: a facile fabrication strategy for freestanding three dimensional silicon micro-and nanostructures by a modified Bosch etch process J. Micromech. Microeng. 28(10) 105012

[10] Chang B, Zhou C, Tarekegne A T, Yang Y, Zhao D, Jensen F, Hübner J and Jansen H 2019 Large Area Three-Dimensional Photonic Crystal Membranes: Single-Run Fabrication and Applications with Embedded Planar Defects Adv. Opt. Mater. 7(2) 1801176

[11] Takahashi S, Suzuki K, Okano M, Imada M, Nakamori T, Ota Y, Ishizaki K and Noda S 2009 Direct creation of threedimensional photonic crystals by a top-down approach Nat. Mater. 8(9) 721

[12] Takahashi S, Okano M, Imada M and Noda S 2006 Threedimensional photonic crystals based on double-angled etching and wafer-fusion techniques Appl. Phys. Lett. 89(12) 123106

[13] Jeong HE, Lee J K, Kim H N, Moon S H and Suh KY 2009 A nontransferring dry adhesive with hierarchical polymer nanohairs Proc. Natl. Acad. Sci. U.S.A. 106(14) 5639-5644

[14] Chang B, Tang Y , Liang M, Jansen H, Jensen F, Wang B, Mølhave K, Hübner J and Sun H 2019 Highly Ordered 3D Silicon Micro-Mesh Structures Integrated with Nanowire Arrays: A Multifunctional Platform for Photodegradation, Photocurrent Generation, and Materials Conversion ChemNanoMat 5(1) 92-100

[15] Chang B, Leussink P, Jensen F, Hübner J and Jansen H 2018 DREM: Infinite etch selectivity and optimized scallop size distribution with conventional photoresists in an adapted multiplexed Bosch DRIE process. Microelectron. Eng. 191, $77-$ 83

[16] Ingram S G 1990 The influence of substrate topography on ion bombardment in plasma etching. J. Appl. Phys. 68(2) 500504

[17] Kubota T, Ohtake H, Araki R, Yanagisawa Y, Iwasaki T, Ono K, Miwa K and Samukawa S 2013 Prediction of etchingshape anomaly due to distortion of ion sheath around a largescale three-dimensional structure by means of on-wafer monitoring technique and computer simulation J. Phys. D. 46(41) 415203

[18] Murakawa S and McVittie J P 1994 Mechanism of surface charging effects on etching profile defects Jpn. J. Appl. Phys. 33(4S) 2184.

[19] Arikado T, Horioka K, Sekine M, Okano H and Horiike Y 1988 Single silicon etching profile simulation. Jpn. J. Appl. Phys. 27(1R) 95

[20] Gottscho R A, Jurgensen C W and Vitkavage D J 1992 Microscopic uniformity in plasma etching J. Vac. Sci. Technol. B 10(5) 2133-2147

[21] Murakawa S, Fang S and McVittie J P 1994 Ion trajectory distortion and profile tilt by surface charging in plasma etching Appl. Phys. Lett. 64(12) 1558-1560

[22] Li L, Zhang C, Tuan C C, Chen Y and Wong C P 2018 High-aspect-ratio microstructures with versatile slanting angles 
on silicon by uniform metal-assisted chemical etching $J$. Micromech. Microeng. 28(5) 055006

[23] Chen H, Wang H, Zhang X H, Lee C S and Lee S T 2010 Wafer-scale synthesis of single-crystal zigzag silicon nanowire arrays with controlled turning angles Nano Lett.10(3) 864-868

[24] Park S S and Ha C S 2018 Hollow mesoporous functional hybrid materials: fascinating platforms for advanced applications. Adv. Funct. Mater. 28(27) 1703814.

[25] Shchukin D G, Sukhorukov G B and Möhwald H 2003 Smart inorganic/organic nanocomposite hollow microcapsules. Angew. Chem. 42(37) 4472-4475 